\title{
Optogenetic stimulation of the motor cortex alleviates neuropathic pain in rats of infraorbital nerve injury with/without CGRP knock-down
}

Jaisan Islam ${ }^{1}$, Elina $\mathrm{KC}^{1}$, Byeong Ho Oh², Soochong Kim³ ${ }^{3}$ Sang-hwan Hyun ${ }^{3}$ and Young Seok Park ${ }^{1,2,3,4^{*}}$ (D)

\begin{abstract}
Background: Previous studies have reported that electrical stimulation of the motor cortex is effective in reducing trigeminal neuropathic pain; however, the effects of optical motor cortex stimulation remain unclear.

Objective: The present study aimed to investigate whether optical stimulation of the primary motor cortex can modulate chronic neuropathic pain in rats with infraorbital nerve constriction injury.

Methods: Animals were randomly divided into a trigeminal neuralgia group, a sham group, and a control group. Trigeminal neuropathic pain was generated via constriction of the infraorbital nerve and animals were treated via selective inhibition of calcitonin gene-related peptide in the trigeminal ganglion. We assessed alterations in behavioral responses in the pre-stimulation, stimulation, and post-stimulation conditions. In vivo extracellular recordings were obtained from the ventral posteromedial nucleus of the thalamus, and viral and a-CGRP expression were investigated in the primary motor cortex and trigeminal ganglion, respectively.

Results: We found that optogenetic stimulation significantly improved pain behaviors in the trigeminal neuralgia animals and it provided more significant improvement with inhibited a-CGRP state than active a-CGRP state. Electrophysiological recordings revealed decreases in abnormal thalamic firing during the stimulation-on condition.

Conclusion: Our findings suggest that optical motor cortex stimulation can alleviate pain behaviors in a rat model of trigeminal neuropathic pain. Transmission of trigeminal pain signals can be modulated via knock-down of aCGRP and optical motor cortex stimulation.
\end{abstract}

Keywords: Optogenetics, Neuropathic pain, Trigeminal ganglion, a-CGRP, Thalamus, Motor cortex

\footnotetext{
* Correspondence: youngseokparkmd@gmail.com;

radiosurgerypark@chungbuk.ac.kr

'Department of Neuroscience, College of Medicine, Chungbuk National

University, Cheongju, South Korea

${ }^{2}$ Department of Neurosurgery, Chungbuk National University Hospital,

Cheongju, South Korea

Full list of author information is available at the end of the article
}

(c) The Author(s). 2020 Open Access This article is licensed under a Creative Commons Attribution 4.0 International License, which permits use, sharing, adaptation, distribution and reproduction in any medium or format, as long as you give appropriate credit to the original author(s) and the source, provide a link to the Creative Commons licence, and indicate if changes were made. The images or other third party material in this article are included in the article's Creative Commons licence, unless indicated otherwise in a credit line to the material. If material is not included in the article's Creative Commons licence and your intended use is not permitted by statutory regulation or exceeds the permitted use, you will need to obtain permission directly from the copyright holder. To view a copy of this licence, visit http://creativecommons.org/licenses/by/4.0/ The Creative Commons Public Domain Dedication waiver (http://creativecommons.org/publicdomain/zero/1.0/) applies to the data made available in this article, unless otherwise stated in a credit line to the data. 


\section{Introduction}

Trigeminal neuralgia (TN) is a facial pain syndrome characterized by paroxysmal pain attacks along the distribution of the trigeminal nerve. Among the two forms (typical and atypical) of TN, most typical cases of TN respond well to surgical treatments such as rhizotomy or, microvascular decompression. However, in case of patients with atypical TN (e.g., TN associated with disease or lesions in the trigeminal sensory system), the condition is often refractory to conventional treatment strategies [1, 2]. Therefore, finding effective alternative treatment approaches are indispensable.

In various chronic pain syndromes, the primary motor cortex (M1) plays a key role in the modulation of pain. Motor cortex stimulation (MCS) was first performed by Tsubokawa et al. in 1991 for the treatment of central pain, yielding promising results. MCS has since been for the treatment of several intractable pain syndromes, including, post-stroke pain, phantom limb pain, pain associated with multiple sclerosis/spinal cord injury pain, and postherpetic neuralgia. Studies have reported that $>$ $75 \%$ of patients with TN achieve good or excellent pain relief following MCS [3-6]. However, both tolerance to MCS and the clinical efficacy of treatment appear to wane over time, ultimately resulting in short-term outcomes $[1,7]$. Hence, further studies are required to determine the true benefits and risks of MCS and to find potential measures which can act as an adjuvant for maintaining a sustainable long-term result after MCS in patients with trigeminal neuropathic pain.

To understand the neural substrates of behavior, functional dissection of neural circuits is crucial. Various approaches have been used to intervene with neural dynamism but via the delivery of specific wavelengths of light following the introduction of genes that encode light-sensitive transmembrane channels, the Optogenetics and it's advancement of strategies has enabled the precise temporal and spatial control of specific neural populations [8, 9]. Several animal studies have reported significant interactions among different brain areas in chronic pain, as well as the effects of therapeutic optogenetics on such interactions. Accumulating evidence has demonstrated that optogenetic stimulation of the brain exerts an impact on both physical and emotional aspects of pain, as it provides better temporal specificity than pharmacologic or electrical interventions [10-12]. Although the precise mechanism underlying its effects remains to be fully elucidated, optogenetic motor cortex stimulation may represent an alternative for patients in whom conventional treatment strategies such as pharmacological or, deep brain stimulation (DBS) have proven to fail to deliver satisfactory outcomes.

Calcitonin gene-related peptide (CGRP) is considered the most important neuropeptide in the trigeminal system, exhibiting an ascendant presence in TRPV1expressing sensory neurons as $\alpha$-CGRP. Studies have revealed that $\alpha$-CGRP plays a key role in the trigeminal nociceptive system, and antibodies directed towards CGRP and CGRP receptor antagonists showing promising effects. Such studies suggest that $\alpha$-CGRP plays a vital role in regulating pain in patients with chronic TN $[13,14]$.

Although effective in many cases, electrical MCS is not without risks and setbacks, necessitating further investigation of alternative treatment strategies such as optical MCS. Thus, in this present study, we utilized a rat model of TN to investigate behavioral changes following optogenetic modulation of neurons in cortical layer $\mathrm{V}$ of M1 in animals with inhibited or active state of $\alpha$-CGRP.

\section{Materials and methods}

\section{Experimental animal and ethical review}

The present study included 72 adult female SpragueDawley rats (age: 8 weeks; Koatech, Pyeongtaek, Korea), each weighing $200-250 \mathrm{~g}$ on arrival. Animals were allowed ad libitum access to fresh food and water and maintained under a 12-h light/dark cycle at a humidity of $50-60 \%$. We used female animals because it has been well established that trigeminal nerve-associated disorders are more prevalent among females than among males, and that CGRP expression is significantly higher in females than in males [13]. All animal tests were conducted in a randomized, double-blind, controlled manner. The animals were randomly divided into a TN group $(n=32)$, a sham group $(n=32)$ and a naive control group $(n=8)$. The timeline of the experimental protocol is shown in Fig. 1. By the Institutional Animal Care and Use Committee (IACUC) of Chungbuk National University (CBNUA-1346-20-02), all experiments were approved and all animal experiments were performed during the light period at the Laboratory Animal Research Center of Chungbuk National University.

\section{Generation of trigeminal neuralgia model}

Ligation of the infraorbital nerve (ION) was performed in 32 animals in accordance with the procedure described by Melanie et al., 2008 [15]. Animals were anesthetized with an intraperitoneal (i.p.) injection of a mixture of $15 \mathrm{mg} / \mathrm{kg}$ Zoletil (Zoletil50 ${ }^{\circ}$, Virbac Laboratories, Carros, France) and $9 \mathrm{mg} / \mathrm{kg}$ Rompun (Rompun ${ }^{\odot}$, Bayer, Seoul, South Korea) in saline, following which they were mounted onto the surgical field in a prone position. The skin above the eye was then shaved, and the animals were placed in a stereotaxic frame. Ophthalmic ointment was applied to the cornea to prevent damage associated with drying of the eyes. A skin incision of approximately $7 \mathrm{~mm}$ in length was made along the curve of the frontal bone in the anterior-posterior direction, 2 

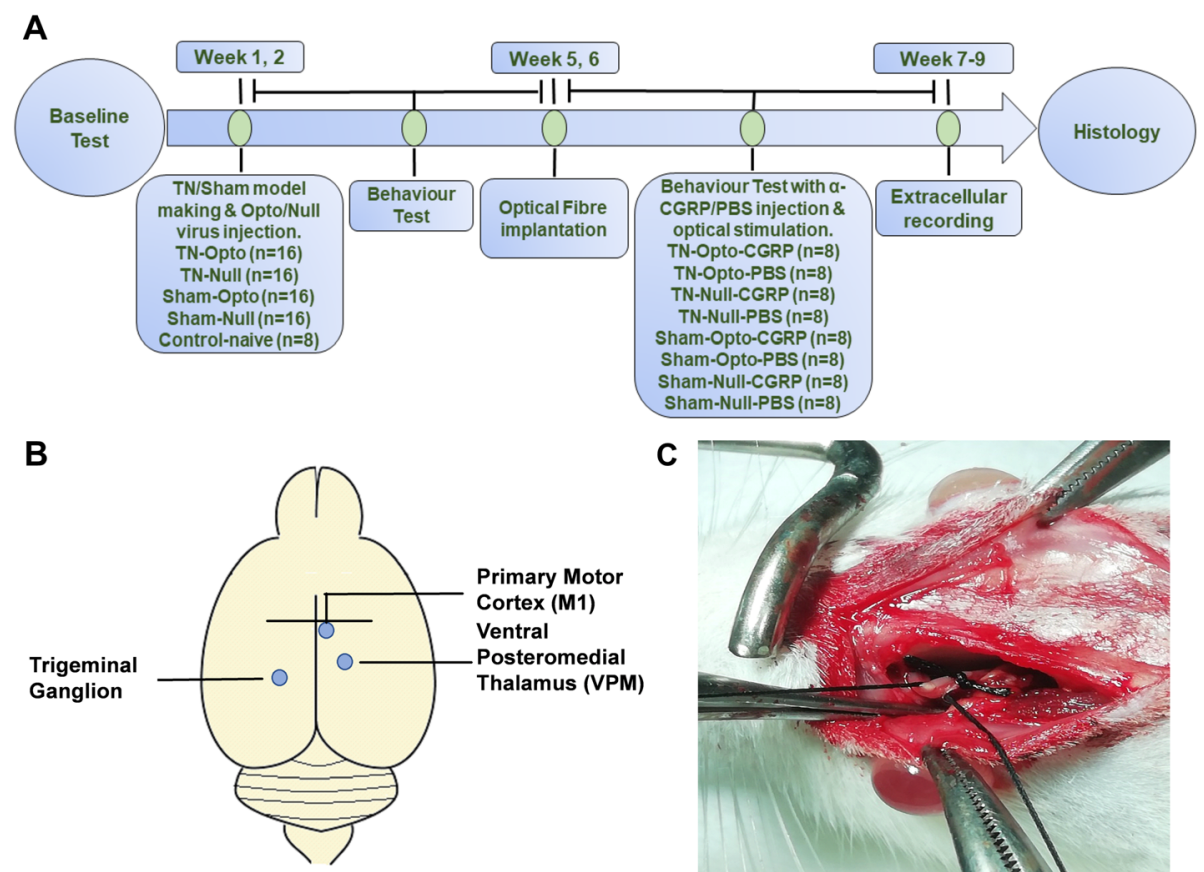

C

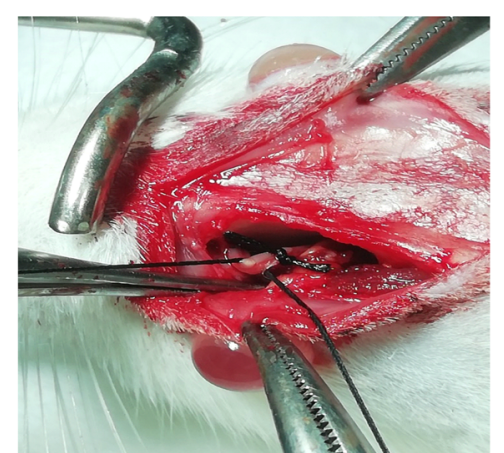

Fig. 1 Experimental animal model and timeline. a Experimental timeline. b Schematic diagram of the experimental animal model and optogenetic virus injection site. C. Ligation of the ION to produce the TN model. ION: infraorbital nerve, TN: trigeminal neuralgia

$\mathrm{mm}$ above the left eye. Moving laterally, the fascia and muscle were gently separated from the bone using a periosteal elevator. The ION could be observed on the maxillary bone following retraction of the eye. After revealing the ION, we prepared for ligature placement by gently freeing approximately $8 \mathrm{~mm}$ of the ION from the surrounding connective tissue. The ION was stretched slightly using a blunt needle with a curved head for ligature placement. The two ligatures were gently placed 3$4 \mathrm{~mm}$ apart, following which they were tightened until the ION was barely constricted. Finally, the incision above the eye was sutured with silk (3-0). Sham animals $(n=32)$ underwent identical procedures, with the exception of ligature placement. After surgery, fresh pelleted food and water were given to the rats ad libitum and checked for survival every day for at least 1 week. Naive animals did not go through above described surgical procedure.

\section{Injection of optogenetic viral vector}

Animals of the TN group were randomly divided into two groups. Sixteen rats were subjected to optogenetic viral vector (AAV2-CaMKII-hChR2-EYFP) injection contralateral to the ION, while the remaining 16 were subjected to null virus (AAV2- CaMKII-EYFP) injection. Vectors were intracranially injected into layer $\mathrm{V}$ of $\mathrm{M} 1$ (AP - $1 \mathrm{~mm}$, ML $1.5 \mathrm{~mm}$, DV - $1.5 \mathrm{~mm}$ [16-18];) under general anesthesia. Animals of the sham group were also divided into two groups for injection of either the optogenetic virus or null virus. We injected an adenoassociated virus carrying the ChR2-EYFP fusion protein under the control of an excitatory neuron-specific CaMKII promoter. Prior to virus injection, anesthesia procedure was performed via an intraperitoneal injection of a mixture of $15 \mathrm{mg} / \mathrm{kg}$ tiletamine/zolazepam (Zoletil50, Virbac Laboratories, Carros, France) and $9 \mathrm{mg} / \mathrm{kg}$ xylazine (Rompun ${ }^{\oplus}$, Bayer, Seoul, Seoul, South Korea). Then, $2 \mu \mathrm{l}$ of virus was injected using a Hamilton syringe and an automatic micro-syringe pump at a rate of $0.3 \mu \mathrm{l} / \mathrm{min}$. Then the needle was kept in the same place after injection for $5 \mathrm{~min}$ to ensure virus absorption, following which it was slowly retracted.

\section{Behavioral testing}

All behavior tests were conducted 1 day before (baseline) and every 7 days after model making. The behavior tests were performed in the following sequence:

\section{Air-puff test}

Animals were placed in a Plexiglas cage $(20 \times 20 \times 14$ $\mathrm{cm}$ ) with a grid floor under quiet condition. Rats were kept in the room for at least $30 \mathrm{~min}$ to get habituated to the environment. Continuous puffs of air (starting from 5 psi (pound per square inch), duration: $4 \mathrm{~s}$ ) were delivered to the ipsilateral side to the ION of the face at intervals of $10 \mathrm{~s}$. Air puffs were delivered through a narrow tubing tip placed approximately $1 \mathrm{~cm}$ from the face at an angle of $90^{\circ}$. The psi was increased over trials 
and during administration of the air puff, we measured and evaluated the psi level of air puff at which aggressive behavioral responses such as biting or turning the head were exhibited by the animal. If the animal exhibited no response to a stimulus of at least $40 \mathrm{psi}$, the test was ended, and the animal was given a break for a few minutes. Mechanical thresholds were assessed and illustrated $>50 \%$ of the overall responses [2].

\section{Mechanical allodynia test}

Orofacial sensitivity to mechanical stimulation was assessed using von Frey filaments. Animals were first acclimated in a Plexiglas cage for $1 \mathrm{~h}$. During this time, von Frey filaments were brought near the animals every $5 \mathrm{~min}$ for habituation. Animals were allowed to explore the filaments for a few seconds before they were removed. Stimuli were administered after the animals had become accustomed to the filament when placed near the center of the vibrissal pad within the territory of ipsilateral ION. The intensity of stimulation was gradually increased. The lowest force (in grams) required to elicit reactions such as immediate withdrawal, attacking the filament by biting or grabbling, escape behaviors, or asymmetric stroking of the face was recorded as the response threshold force [19].

\section{Facial cold hyperalgesia test}

Animals were placed in the same Plexiglas cage described above. For this test, a few drops of acetone were placed on the vibrissal pad ipsilateral to the ION using a glass syringe, following which we counted the number of scratching/rubbing behaviors over the next $2 \mathrm{~min}$. Body parts other than the face were excluded for this assessment [2].

\section{Optic fiber implantation}

Four weeks after optogenetic virus inoculation, animals were positioned in a stereotactic frame following induction of anesthesia. An optic fiber was implanted into the skull contralateral to the ION (AP: $-1 \mathrm{~mm}$, ML: 1.5 $\mathrm{mm}$ ) for the transmission of laser pulses to layer $\mathrm{V}$ of M1. Optic fibers $(200 \mu \mathrm{m}$ core, $230 \mu \mathrm{m}$ outer diameter, numerical aperture of 0.48 , hard polymer cladding type, Doric Lenses; Québec City, Québec, Canada) were cut to a length of $1.4 \mathrm{~mm}$ to optimize targeting of M1. Dental cement was used to fix the fiber firmly in place (Orthoject Pound Package, Lang Dental, USA).

\section{Selective knock-down of a-CGRP in trigeminal ganglion}

Before performing the behavioral tests and extracellular recording with optic stimulation, animals in each group (TN-Opto, TN-Null, Sham-Opto, and Sham-Null) were again divided into two sub-groups of 8 animals each. Animals in one group received an injection of $\alpha$-CGRP
( $\alpha$-CGRP (8-37) (mouse, rat) trifluoroacetate salt, $1 \mathrm{mg} /$ $\mathrm{ml}$, BACHEM) as a CGRP receptor antagonist, while animals in the other group received an injection of PBS. Animals of CGRP group received an intravenous injection of $10 \mu \mathrm{l}$ of $\alpha$-CGRP $10 \mathrm{~min}$ prior to the behavior test. In case of extracellular recording, $\alpha$-CGRP was injected directly into the ipsilateral trigeminal ganglion (AP: $-3.5 \mathrm{~mm}$, ML: $-3.6 \mathrm{~mm}$, DV: $-12 \mathrm{~mm}$ ) [20] and the recording was performed after that. $\alpha$-CGRP was injected using a Hamilton syringe and an automatic micro-syringe pump at a rate of $0.3 \mu \mathrm{l} / \mathrm{min}$. After injection, the needle was kept in the same place for $5 \mathrm{~min}$ to ensure absorption of CGRP, following which it was slowly retracted.

\section{Optical stimulation}

We used a laser power supply with a wavelength of $473 \mathrm{~nm}$ (ADR-700D, Shanghai, China) and a waveform generator (Keysight 33511b-CFG001, Keysight, Santa Rosa, CA, USA) to regulate the waveform and pulse width of the laser (Fig. 2). The laser's intensity was set to $10 \mathrm{~mW}$, the pulse width was set to $4 \mathrm{~ms}$, the pulses were set to $20 \mathrm{~Hz}$ and the duration of stimulation was $5 \mathrm{~min}$ [21]. Behavioral differences were examined in animals inoculated with the optogenetic virus (pre, stim, post) to determine the effects of optical neuromodulation.

\section{In vivo extracellular recording}

Six weeks after ligation of the ION, rats were anesthetized with $15 \mathrm{mg} / \mathrm{kg}$ tiletamine/zolazepam and $9 \mathrm{mg} / \mathrm{kg}$ xylazine to prepare for extracellular recordings. Extracellular recordings were obtained from the VPM (AP -3.5 $\mathrm{mm}, \mathrm{ML} 2.8 \mathrm{~mm}, \mathrm{DV}-6 \mathrm{~mm}$ ) [2] using a single electrode. Following a 20-min resting condition, thalamic neuronal spikes and firing rate were recorded during the pre-stimulation, stimulation (blue: $473 \mathrm{~nm}$ ), and poststimulation states in vivo. A glass-insulated carbon fiber microelectrode (Cat. No.: E1011-20, Carbostar-1, Kation Scientific, LLC, MN 55414 USA) was used for recording in the thalamus. Recordings were obtained for $5 \mathrm{~min}$ during each stage, with a 5-min gap between stages. We picked well-isolated clusters and recording of neuronal signals was performed using a Digital Lynx SX (Neuralynx, Bozeman, USA) data-acquisition system along with Cheetah software. We digitized and bandpass filtered at $40 \mathrm{kHz}$ and $1 \mathrm{~Hz}-5 \mathrm{kHz}$, respectively. We sorted offline using Spike Sorter 3D (Neuralynx Inc., Montana, USA). Neuronal discharge was evaluated in the contralateral VPM of TN model and sham animals. Analysis of the rate histograms (spikes/s) of lesioned animals under different optical conditions was accomplished using Neuroexplorer (Neuralynx Inc., Montana, USA). 


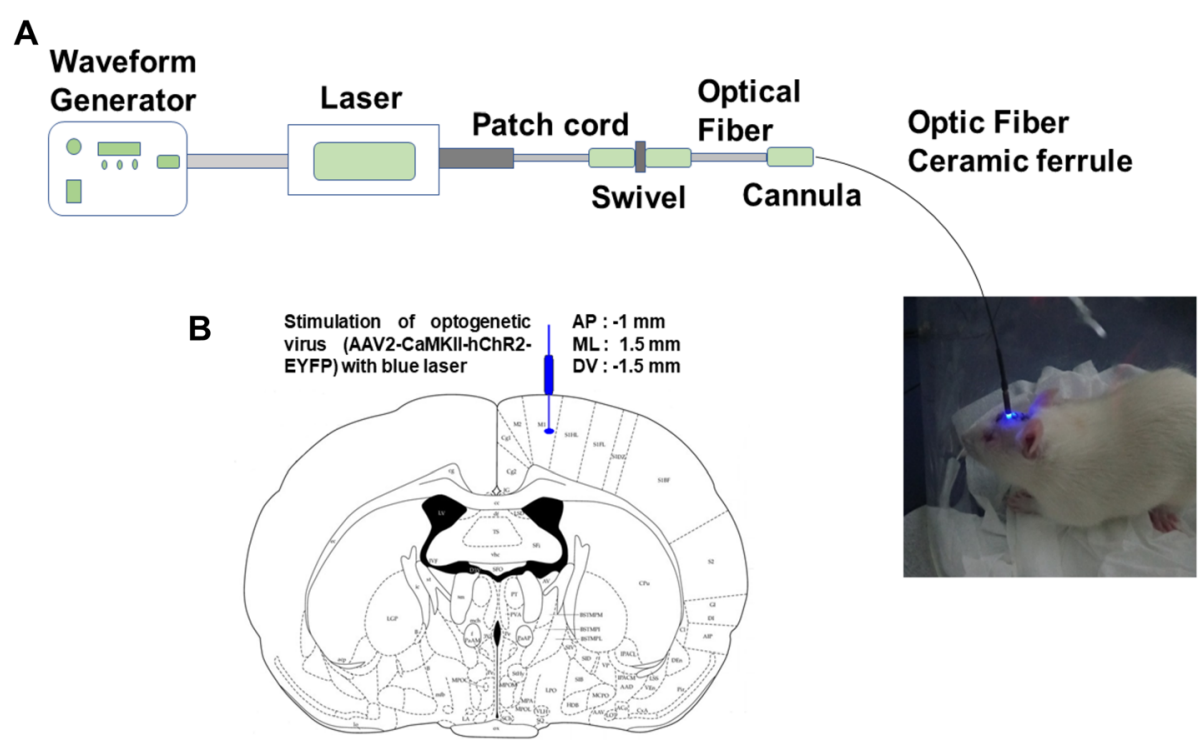

Fig. 2 Optogenetic stimulation procedure. a Complete setup of optic stimulation. b Optic fiber implantation. c Stimulation of primary motor cortex using a blue laser

\section{Histological examinations}

The rats were deeply anesthetized and transcardially perfused with PBS followed by $4 \%$ paraformaldehyde. The brains and trigeminal ganglion were extracted and fixed overnight in the same post fixed solution, followed which they were dehydrated in $30 \%$ sucrose solution.

We embedded brains and trigeminal ganglions in optimal cutting temperature (O.C.T.) compound (Tissue Tek $^{\oplus}$ - Sakura, USA), following which they were cryopreserved with liquid nitrogen and isopentane at $-79^{\circ} \mathrm{C}$. A cryostat (Thermo Scientific, Waltham, MA, USA) was used to cut coronal sections of the brain $(20 \mu \mathrm{m})$ and trigeminal ganglion $(10 \mu \mathrm{m})$. The brain sections were incubated with DAPI and mounted with coverslips for examination under fluorescence microscope. To observe the action of $\alpha$-CGRP receptor antagonist in trigeminal ganglion, the animals were sacrificed immediately after the extracellular recording and the trigeminal ganglion was collected. Trigeminal ganglion sections were immunostained with anti-CGRP antibody (1:200, ab36001, Abcam), following which they were incubated in serum block solution for $1 \mathrm{~h}$ and with anti- $\alpha$ CGRP antibody overnight. The corresponding secondary antibody was applied prior to staining with DAB (Vector Laboratory, California, USA). Nuclei were counterstained with hematoxylin. Finally, the sections were dehydrated and mounted with coverslips and examined under a microscope.

\section{Analysis of the bursting and firing rates}

Activity in thalamic neurons was expressed as overall firing rates (spikes/s) in each optical stimulation condition using NeuroExplorer software (Neuralynx Inc.). Activity was assessed for $5 \mathrm{~min}$ in each state: pre-stimulation, stimulation, and post-stimulation. We defined burst rates as a group of at least three spikes with a maximum interval of $4 \mathrm{~ms}$ between spikes, with an interval of 100 ms between bursts. We selected similar inter-spike interval histograms for comparison among the groups.

\section{Statistical analysis}

Data were analyzed using GraphPad Prism (GraphPad Software version 8.4.2, Inc., San Diego, CA, USA) and represented as the mean \pm standard deviation (SD). We performed either an unpaired $t$-test, two-way analysis of variance (ANOVA) with Tukey's post hoc test, or a repeated-measures ANOVA depending on the conditions of the experiment. Behavioral tests were assessed based on the mean values for each of the three optical states. Unpaired $t$-tests were used to compare firing rates between TN and sham-operated animals. All the statistical data were measured as significant at $p<0.005$.

\section{Results}

Changes in pain behaviors following ION ligation

Following ION ligation, behavioral test scores suggested the presence of chronic pain in $\mathrm{TN}$ animals, whereas no significant changes in behavioral scores were observed in sham and control animals. In TN group, a two-way ANOVA revealed significant, gradual decreases in airpuff test scores over the 4 weeks $(22.46 \pm 1.68$ psi to $12.17 \pm 1.62$ psi; $F(2,165)=180.1, p<0.001$; Fig. 3a). In addition, facial cold hyperalgesia scores significantly increased from $14.90 \pm 1.64$ to $24.85 \pm 1.76$ (two-way 

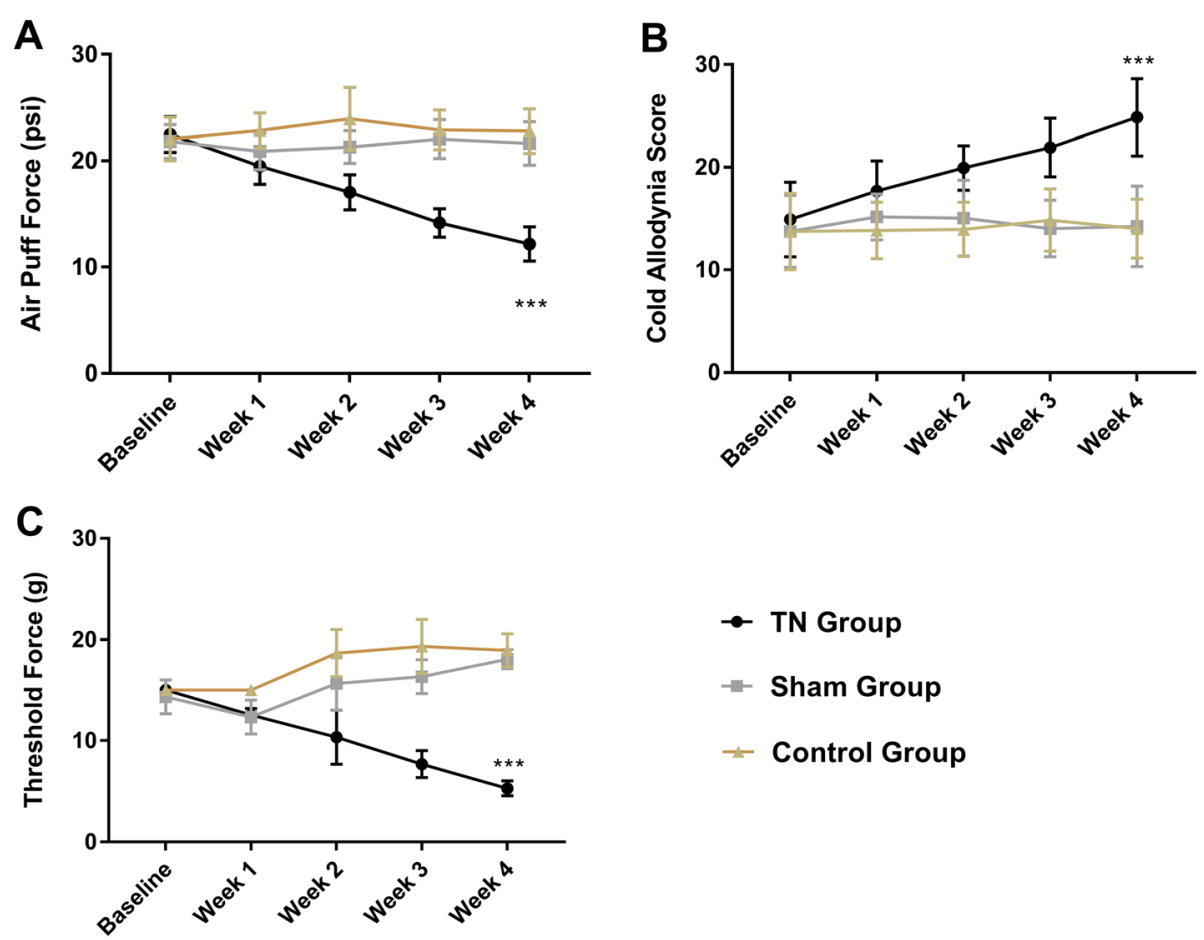

Fig. 3 Behavioral test results for the TN, sham, and control groups. a Air-puff test results for the ipsilateral trigeminal facial area. b Cold hyperalgesia results for the ipsilateral facial area following treatment with acetone drops. $\mathbf{c}$ Mechanical allodynia (von Frey test) results for the ipsilateral facial side. ${ }^{* *}, p<0.001$, significant difference determined via an analysis of variance (ANOVA)

ANOVA, $F(2,165)=48.59, p<0.001$; Fig. 3b). Mechanical allodynia scores also significantly decreased from $15 \pm 00 \mathrm{~g}$ to $5.27 \pm 0.73 \mathrm{~g}$ following surgery $(F(2,165)=$ 259.9, $p<0.001$; Fig. 3c).

\section{Attenuation of hyperalgesia via a-CGRP injection and optogenetic stimulation}

Optical modulation induced significant changes in behavioral scores in the TN group. Our findings indicated that optogenetic stimulation resulted in neuromodulation of cells in layer V of M1. TN-Opto-CGRP animals exhibited significant alterations of behavioral responses in air-puff test during the stimulation-ON condition. For these animals, a two-way ANOVA revealed a significant effect of optogenetic modulation (pre-stimulation: $11.523 \pm 2.13 \mathrm{psi}$; stimulation $\mathrm{ON}$ : $18.16 \pm 1.78 \mathrm{psi}$; poststimulation: $16.045 \pm 2.44 \mathrm{psi} ; F(2,24)=36.65, p<0.01)$ (Fig. 4A(a)). Significant decreases in cold hyperalgesia were also observed during the $\mathrm{ON}$ condition (pre-stimulation: $23.5243 \pm 2.9854$; stimulation $\mathrm{ON}$ : $16.563 \pm 3.76$; post-stimulation: $18.943 \pm 2.2476$; two-way ANOVA, $F$ $(2,24)=39.55, p<0.01)($ Fig. 4B(a)). Mechanical allodynia scores also improved in TN-Opto-CGRP animals during the $\mathrm{ON}$ condition (pre-stimulation: $4.1 \pm 0.8 \mathrm{~g}$; stimulation $\mathrm{ON}$ : $8.9 \pm 0.9 \mathrm{~g}$; post-stimulation: $6.8 \pm 0.7 \mathrm{~g}$; two-way ANOVA, $F(2,24)=24.21, p<0.05$ ) (Fig. $4 C(a))$.
TN-Opto-PBS animals also exhibited significant changes in behavioral responses due to optogenetic stimulation, including increases in air-puff test scores (pre-stimulation: $11.10 \pm 3.13 \mathrm{psi}$; stimulation $\mathrm{ON}$ : $15.81 \pm 2.68$ psi; post-stimulation: $13.45 \pm 2.54$ psi; twoway ANOVA, $F(2,24)=22.673, p<0.05$ ) (Fig. $4 \mathrm{~A}(\mathrm{~b}))$. These animals also exhibited significant decreases in cold hyperalgesia during the $\mathrm{ON}$ condition (pre-stimulation: $23.345 \pm 2.11$; stimulation $\mathrm{ON}$ : $19.13 \pm 1.53$; post-stimulation: $20.711 \pm 2.46$; two-way ANOVA, $F(2,24)=25.88, p<0.05)$ (Fig. 4B(b)). In addition, we observed improvements in mechanical allodynia scores in the TN-Opto-PBS group during the ON condition (pre-stimulation: $3.25 \pm 0.64 \mathrm{~g}$; stimulation $\mathrm{ON}$ : $6.15 \pm 0.75 \mathrm{~g}$; post-stimulation: $4.90 \pm$ $0.48 \mathrm{~g}$; two-way ANOVA, $F(2,24) 22.03, p<0.05)$ (Fig. $4 \mathrm{C}(\mathrm{b}))$. The other animal groups (TN-NullCGRP, TN-Null-PBS, Sham-Opto-CGRP, Sham-OptoPBS, Sham-Null-CGRP, Sham-Null-PBS) exhibited no significant changes in behavior due to optogenetic modulation or null virus injection (Fig. $4 \mathrm{~A}(\mathrm{c}-\mathrm{h}), \mathrm{B}(\mathrm{c}-$ h), C(c-h)).

We further evaluated the extent of improvement in animals by optic stimulation along with or without $\alpha$ CGRP knock down and it was found that TN-OptoCGRP grouped animals showed more improvement than TN-Opto-PBS group (Fig. 4d). 

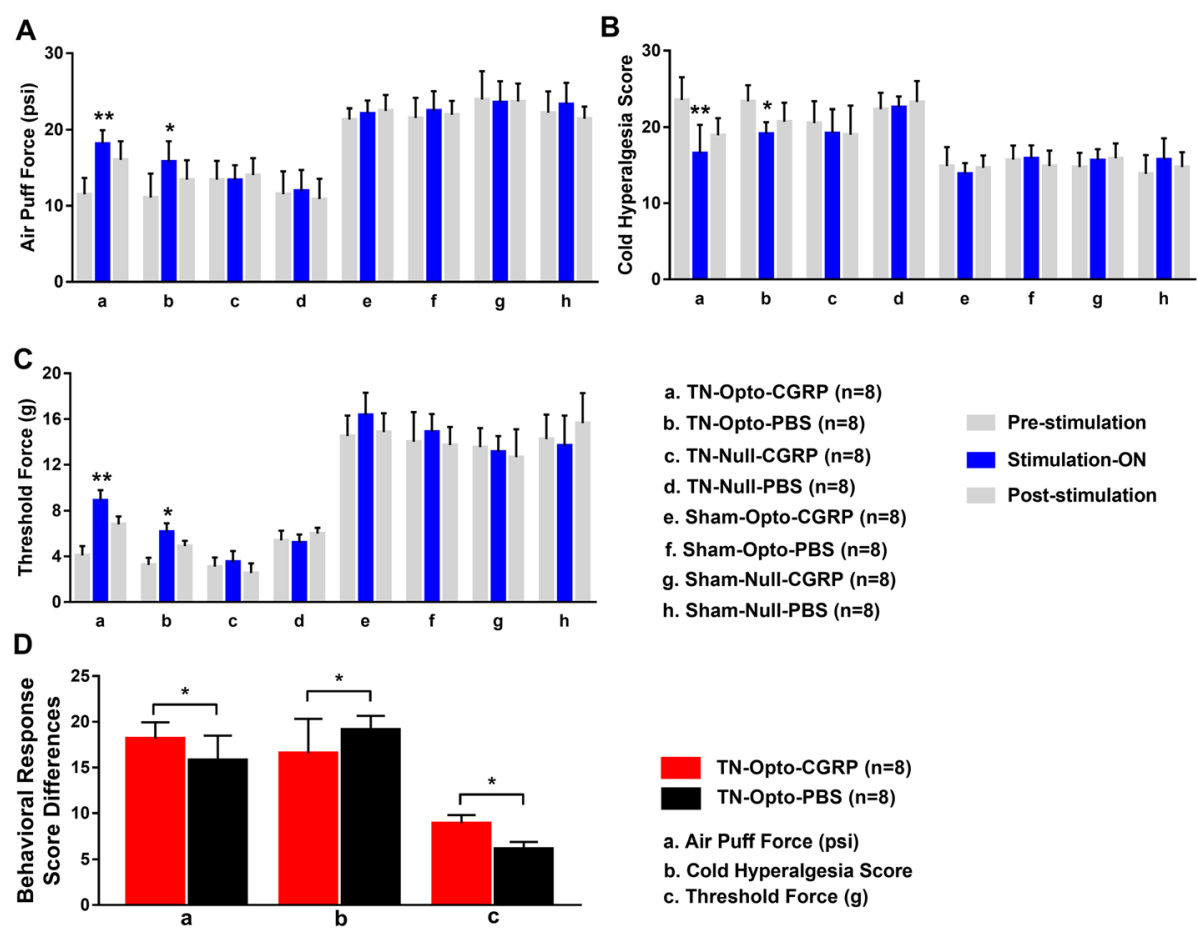

h. Sham-Null-PBS $(n=8)$

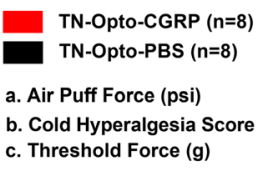

Fig. 4 Changes in behavioral responses following blue laser stimulation (a-c) along with knocking down of a-CGRP (d). Results for the air-puff test (a), cold hyperalgesia test (b), and von Frey test (c) for all eight animal groups. Only the TN-Opto-CGRP (a) and TN-Opto-PBS groups (b) exhibited significant changes in behavioral scores during the stimulation ON condition. No significant changes in behavior were observed in other animal groups during the stimulation ON condition. $\mathbf{d}$ Animals with a-CGRP injection exhibited more improvement in behavioral tests than animals with PBS injection. a Air puff force differences, $\mathbf{b}$ Cold hyperalgesia score differences and (c) Mechanical allodynia score differences between TN-Opto-CGRP and TN-Opto-PBS group. ${ }^{*}, p<0.05 ;{ }^{* *}, p<0.01$, significant difference determined via an analysis of variance (ANOVA)

These findings support our hypothesis that stimulation of neurons in layer V of M1 exerts an antinociceptive effect in $\mathrm{TN}$.

\section{In vivo extracellular recording data}

To confirm whether changes in neuronal activity had occurred, we examined firing rates in VPM neurons in the TN, sham, and control groups. Significantly higher firing rates were observed in TN-lesioned rats (33.435 \pm 5.233 spikes/s) than in sham rats $(22.563 \pm 4.534$ spikes/s, unpaired $t$-test $(\mathrm{t}, \mathrm{df})=(8.882,62), p<0.001)$ or control rats $(21.564 \pm 4.563$ spikes $/ \mathrm{s}$, unpaired $t$-test $(\mathrm{t}, \mathrm{df})=(5.87$, 38), $p<0.001$ ) (Fig. 5a).

We assessed the effects of optical stimulation on M1 in the TN-Opto and TN-Null groups using in vivo extracellular recordings. Optogenetic stimulation induced significant changes in VPM neuron activity in the TN-Opto group. Spike rates in the pre-stimulation, stimulation $\mathrm{ON}$, and post-stimulation conditions in the TN-Opto-CGRP group were $27.66 \pm 4.19,20.85 \pm 2.99$, and $22.09 \pm 2.73$, respectively. A two-way ANOVA revealed significant effects of optic stimulation $(F(2$, $345)=59.09, p<0.01$ ) (Fig. 5b). Spike rates for the prestimulation, stimulation $\mathrm{ON}$, and post-stimulation conditions in the TN-Opto-PBS group were $32.80 \pm 5.28$, $26.45 \pm 4.35$, and $28.03 \pm 3.33$, respectively $(F(2,345)=$ 37.53, $p<0.05$ ) (Fig. 5c). No significant changes in thalamic firing rates were observed in either the TN-NullCGRP or TN-Null-PBS groups (Fig. $5 \mathrm{~d}$ and e).

Burst rates appeared to decrease during optogenetic stimulation in the TN-Opto group. Thalamic burst rates for the pre-stimulation, stimulation $\mathrm{ON}$, and poststimulation conditions in the TN-Opto-CGRP group were $0.44 \pm 0.08 / \mathrm{s}, \quad 0.28 \pm 0.06 / \mathrm{s}$, and $0.40 \pm 0.10 / \mathrm{s}$. respectively. A two-way ANOVA revealed significant effects of optogenetic stimulation $(F(2,276)=5.45, p<$ 0.05 ) (Fig. 6a). Optogenetic stimulation also significantly decreased burst rates in the TN-Opto-PBS group. Thalamic burst rates for the pre-stimulation, stimulation $\mathrm{ON}$, and post-stimulation conditions in the TN-OptoPBS group were $0.75 \pm 0.07 / \mathrm{s}, 0.58 \pm 0.06 / \mathrm{s}, 0.67 \pm 0.10 / \mathrm{s}$, respectively. A two-way ANOVA revealed significant effects of optogenetic stimulation $(F(2,276)=3.57, p<$ 0.05 ) (Fig. 6a). However, stimulation induced no significant changes in burst rates in the TN-Null-CGRP and TN-Null-PBS groups. In both the TN-Opto-CGRP and TN-Opto-PBS groups, we observed greater inhibition of thalamic responses during the stimulation $\mathrm{ON}$ state than 


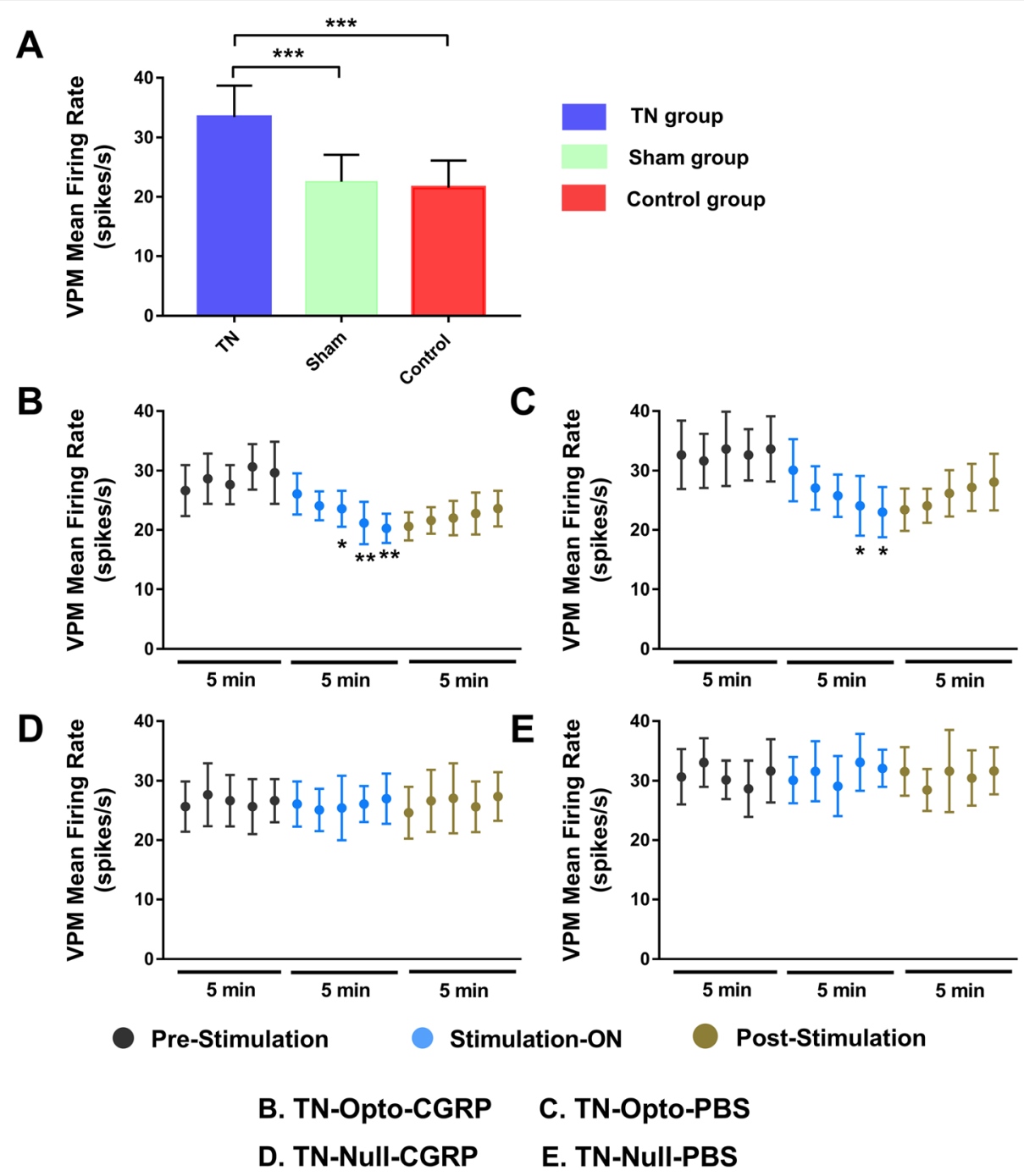

Fig. 5 Electrophysiological findings for VPM thalamic output due to optogenetic stimulation of the motor cortex. a Evoked firing rates in VPM neurons in TN animals versus sham and control animals (**, $p<0.001$, significant difference determined using unpaired $t$-tests). $\mathbf{b}, \mathbf{c}$ In vivo recordings of the VPM thalamus from TN-Opto animals. b Findings for the TN-Opto-CGRP group. $\mathbf{c}$ Findings for the TN-Opto-PBS group. Decrease in firing output (spikes/s) were observed during the stimulation ON period. d, e No changes in firing rates of VPM thalamus were observed in the TN-Null-CGRP and TN-Null-PBS groups, respectively (two-way analysis of variance (ANOVA) *, $p<0.05 ;{ }^{*}, p<0.01$

during the stimulation OFF state (Fig. $6 \mathrm{~b}$ and c). Instantaneous firing frequencies also decreased during the stimulation ON condition (Fig. 6d and e).

These results suggest that optogenetic stimulation of M1 can attenuate thalamic discharge in TN rats. Optogenetic MCS showed significant improvement in pain response, though along with knock down of $\alpha$-CGRP it provided more improved and long-term pain relief. No effects of laser stimulation on thalamic firing patterns were observed in the TN-Null-CGRP and TN-Null-PBS grouped animals.

Viral expression in motor cortex and inactivation of $a-$ CGRP in trigeminal ganglion

The optogenetic target for the present study was layer $\mathrm{V}$ in $\mathrm{M} 1$, which was stereotaxically located using a rat atlas. We observed expression of the optogenetic and null viruses in the contralateral M1 using immunofluorescence (Fig. 7a-f). By using a fluorescence microscope and ImageJ software, viral expression in neurons stained using EYFP and DAPI were obtained. We observed the presence of anti-CGRP antibody binding in trigeminal ganglion cells in animals injected with PBS and antiCGRP binding was absent in $\alpha$-CGRP injected animals (Fig. 7g-l).

\section{Discussion}

In the present study, we aimed to investigate whether optical stimulation of contralateral M1 can modulate chronic neuropathic pain in rats with ION injury. Here we presented results showing that such stimulation 

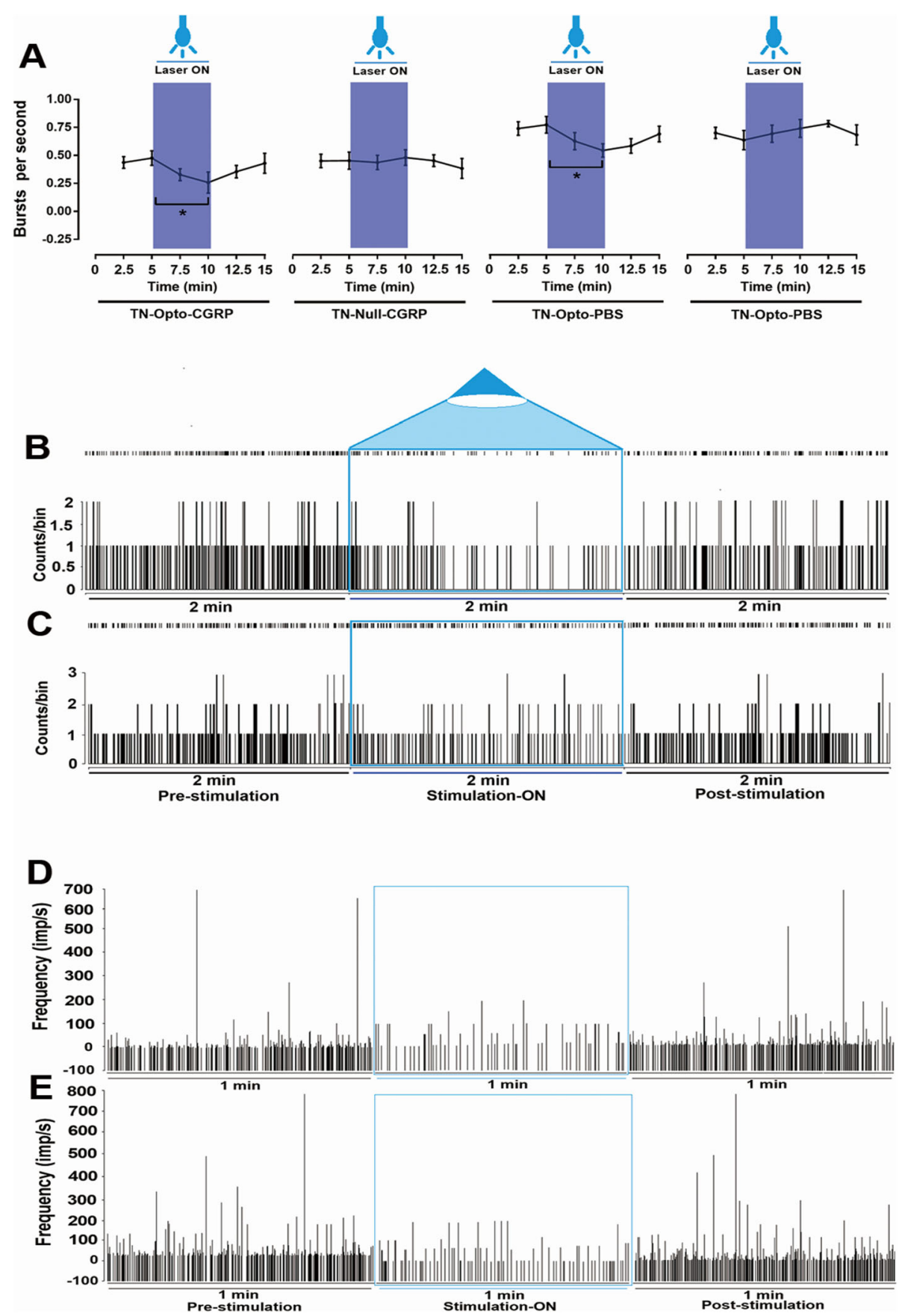

Fig. 6 Burst firing rates in TN animals following optogenetic stimulation (a). Significant changes were observed in the TN-Opto-CGRP and TNOpto-PBS groups. *, $p<0.05$; **, analysis of variance (ANOVA). b, c Peri-event raster histogram of VPM neuron responses in the TN-Opto-CGRP and TN-Opto-PBS groups, respectively. VPM firing rates decreases during optical stimulation. Bin size $=50 \mathrm{~ms}$. d, e Instantaneous firing frequency for VPM neurons in TN-Opto-CGRP and TN-Opto-PBS rats, respectively

significantly improved behavioral responses to pain and modulated thalamic output. Animals with TNP exhibited pain relief responses during MCS by optogenetic system in both with and without $\alpha$-CGRP knockdown condition. In vivo extracellular recording results proved decreased thalamic firing rates while $\mathrm{TN}$ animals were subjected to optogenetic stimulation. All these findings provide foundation for the effectiveness of optogenetic MCS in the treatment of TNP.

TN is the most common form of cranial neuralgia. CCI-ION is a common method of mimicking TN neuropathic pain in animal models. Onset of TN can be 


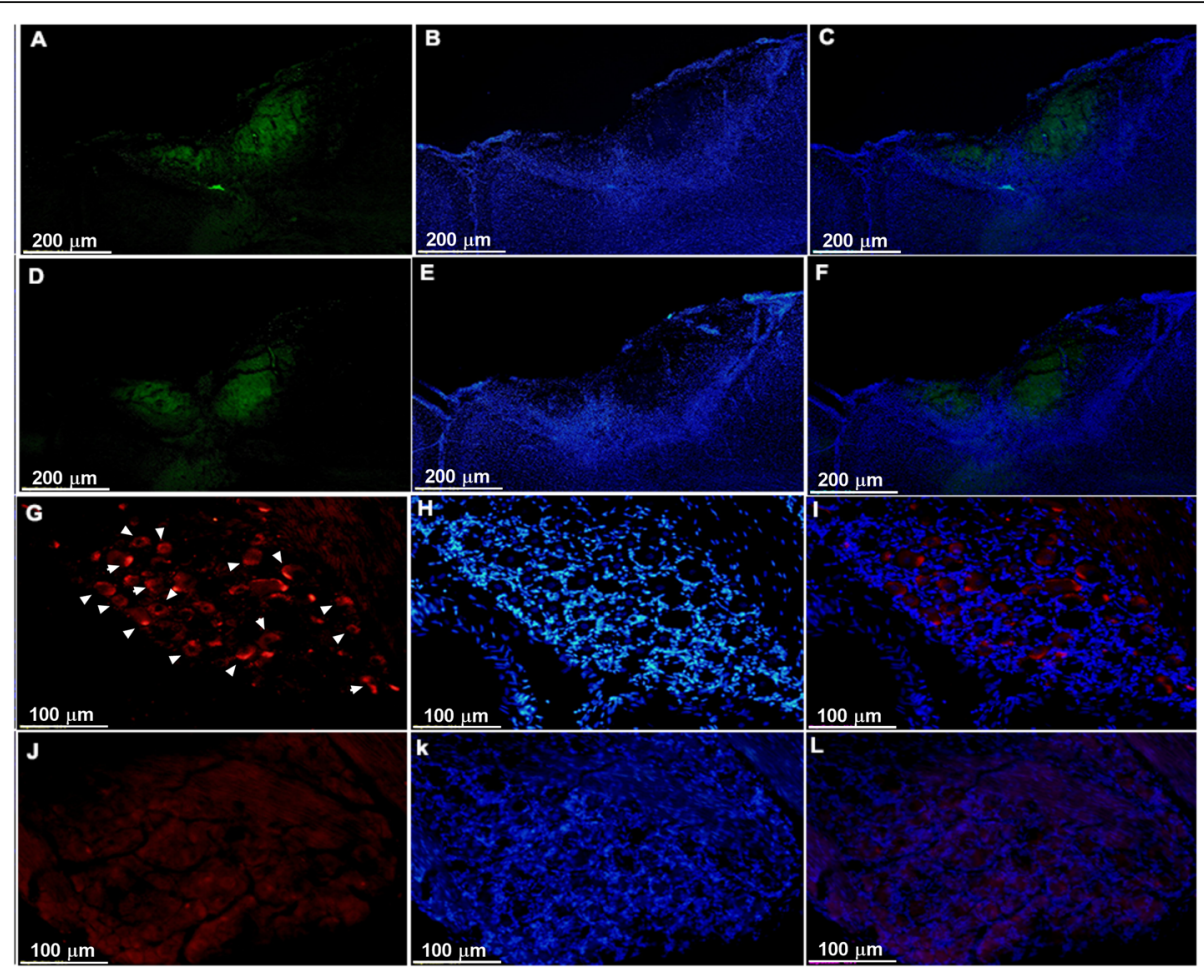

Fig. 7 Immunofluorescence results for viral expression in the primary motor cortex (a-f) and CGRP neuron expression in the trigeminal ganglion $(\mathbf{g}-\mathbf{I})$. $\mathbf{a}, \mathbf{b}, \mathbf{c}$ Optogenetic viral expression in the motor cortex of TN-Opto animals. $\mathbf{d}, \mathbf{e}, \mathbf{f}$ Null viral expression in the motor cortex of TN-Null animals. a, d EYFP, b, e DAPI, and c, f Merge. Scale bar $=200 \mu \mathrm{m}$. g-I PBS and a-CGRP-injected and DAPI-stained trigeminal ganglion cells. $\mathbf{g}-\mathbf{i}$ Presence of anti-CGRP antibody binding due to active state of a-CGRP neuron (arrow) within the trigeminal ganglion of PBS injected animal (j-l). a-CGRP injected and DAPI-stained trigeminal ganglion cells showing absence of CGRP neurons due to inhibition of a-CGRP neurons within the trigeminal ganglion. $\mathbf{g}, \mathbf{j}$ CGRP, $\mathbf{h}, \mathbf{k}$ DAPI, and $\mathbf{i}$, I Merge. Scale bar $=100 \mu \mathrm{m}$

triggered by certain factors such as nerve compression by intracranial and extra cranial tumors or vascular anomalies, demyelinating conditions, nerve trauma, or infectious agents. These conditions can decrease the excitability threshold of affected fibers, thereby promoting improper propagation towards surrounding fibers. Trigeminal afferents project to the mesencephalic nucleus, the principle sensory nucleus of the trigeminal nerve, and the spinal nucleus of the trigeminal nerve by means of the trigeminal ganglion. While non-painful sensory input projects to the principle sensory nucleus, nociceptive information travels down to the spinal nucleus of the trigeminal nerve. These mutually exclusive sensory signals are integrated in the sensory area of the thalamus (i.e., the VPM). In patients with TN, aberrant sensory information leads to the transmission of inappropriate sensory signals to the thalamus, initiating the sensation of pain and changes in behavior [22-27], as observed in our study that after making CCI-ION rat model, animals showed painful behavioral responses and behavior test's scores were significantly different from sham and control grouped animals.

The function of the trigeminal system is regulated by numerous factors including neuropeptides. CGRP is believed to be a leading contributor to the neurogenic inflammation underlying various disorders involving craniofacial structures. Research has indicated that $\alpha$-CGRP neurons are necessary for neuropathic hypersensitivity to mechanical, cold, and heat stimuli; spontaneous pain; and sensitization of mechanical currents in afferent somata. The release of CGRP and other excitatory neurotransmitters from TG neurons in the trigeminal nucleus caudalis (TNC) can excite second-order neurons in the TNC, directly contributing to central sensitization, allodynia, and hyperalgesia. Thermal hyperalgesia is significantly inhibited in $\alpha$-CGRP knockout mice. Furthermore, chemical ablation of $\alpha$-CGRP neurons suggests that they are essential for the detection of cold and heat, and for heat hypersensitivity after both neuropathic and inflammatory injuries. Additional studies have reported that treatment with a CGRP antagonist decreases injuryinduced mechanical hypersensitivity [13, 14, 28-31]. The findings of the present study are in accordance with previous observations of improvements as animal with $\alpha$-CGRP inhibition following optic stimulation showed better improvement in hypersensitivity than animals treated with optical stimulation alone. 
M1 exhibits wide connections to diverse areas of the brain and many spinal efferent and afferent fibers that modulate the transmission of signals associated with painful stimuli. Thus, stimulation of M1 can initiate changes in other brain areas. One advantage of MCS relative to other neurostimulation techniques such as DBS or SCS is that MCS-induced analgesia is not associated with paresthesias [32-34]. By activating the topdown controls associated with intracortical horizontal fibers, MCS exerts its effects which can elicit paresthesias via the activation of non-nociceptive third- or fourthorder somatosensory neurons within the thalamic nucleus [27, 35-37]. The motor cortex is composed of six layers, each playing a distinct role in cerebral function. Among them, layer II/III axons usually send excitatory inputs to pyramidal neurons in layers V and VI. Two classes of projection neurons are important for motor control: corticospinal and corticostriatal neurons. Excitation of layer V neurons due to MCS appears to trigger activation in the striatum, in turn causing rapid phasic alterations in the thalamus by means of GABAergic neurons [38, 39]. Several PET and fMRI studies have indicated that MCS is accompanied by increased blood flow and activation of pain-related brain regions associated with endogenous opioid secretion. These regions lie adjacent to outer brain areas (e.g., orbitofrontal cortex) as well as remote inner brain structures including the insula; anterior, middle, and posterior cingulate cortices; putamen; thalamus; and portions of the brainstem (e.g., PAG and pons) [40-43]. Neuromodulation of M1 may also alter activation at NMDA/AMPA receptors and concentrations of GABA/glutamate at synapses in S1, S2, the thalamus, brainstem, ACC, prefrontal cortex, and other structures. Moreover, studies involving experimental models of neuropathic pain suggest that MCSinduced antinociceptive effects involve the rostroventromedial medulla as well as descending serotoninergic pathways [44-49].

In the present study, we utilized optogenetic techniques to stimulate $\mathrm{M} 1$ in order to induce circuit-specific neuromodulation of neuronal activity in layer V of M1 [10, 50, 51]. To regulate cellular activity with high spatiotemporal resolution, we relied on heterologous expression of the photosensitive actuator channelrhodopsin2 (ChR2), which enabled exact quantitative linking between optical excitation and activation of neurons [52-54]. Since ChR2 is genetically targetable, its expression is used as a powerful tool for increasing cytoplasmic $\mathrm{Ca}^{2+}$ concentrations or depolarizing the cell membrane $[55,56]$. These channels open when activated by blue light $(\sim 473 \mathrm{~nm})$ and were used to induce neuronal excitation in the present study $[57,58]$. CaMKII is a glutamatergic, neuron-specific promoter that drives ChR2 expression. As a result, expression of ChR2 is specific to the excitatory CaMKII $\alpha$-expressing cortical neuron population. As a result, the optical neural interface selectively activates excitatory neurons in the cortex. Our behavioral findings also support the notion that optogenetic MCS improves hypersensitivity by exerting antinociceptive effects as animals with optogenetic virus showed significant improvement than animals with null virus.

Our electrophysiological analyses revealed a robust effect of optical MCS on thalamic and mesencephalic neurons, which may explain the observed analgesic effects. The data we got from extracellular recording of $\mathrm{TN}$ Opto-CGRP and TN-Opto-PBS grouped animals demonstrated significant changes of VPM thalamic responses. Among several structures, the thalamus receives pain signals from various pain pathways. Following peripheral nerve injury, changes in the rhythmic burst firing of thalamic neurons may contribute to the development of chronic pain. The somatotopic ordination of primary afferents found in each brainstem subnucleus is conserved by the trigemino-VPM pathway. The spinal trigeminal nucleus relays somatosensory orofacial information from the brainstem to the thalamus. Spinoand trigemino-thalamic fibers run through the caudorostral extent of the posterior triangular thalamic nucleus and then expand in the Po and VPM. The major trigeminal course to the somatosensory cortex is cognizant to project through the VPM and part of the Po. In accordance with our findings, several previous studies have reported electrophysiological hyperresponsiveness of the contralateral VPM in CCI-ION rats [2, $42,47,48,59-63]$. Optical stimulation of ChR2transfected pyramidal neurons in layer V of M1 inhibits sensory inputs in the spinothalamic tract and modulates cortico-striatal neural circuitry, thereby reducing abnormal thalamic firing. Furthermore, thalamic burst rates during optogenetic stimulation also showed to be decreased in TN-Opto-CGRP and TN-Opto-PBS group.

There have been some limitations in our study. The post-operative brunt may have impact on chronic state of pain as we did not give any medications after surgical intervention. We did not examine the effects of other cortical structure's stimulation. The use of anesthesia may have influenced rates of spontaneous thalamic discharge. We tried as much as possible to avoid the effect of stimulation with Von Frey filaments during neural recording. Furthermore, although we performed electrophysiological analyses of VPM activity in response to pain, we did not perform such analyses in the trigeminal ganglion.

Future studies could utilize the novel technique to determine different dopaminergic neuron's distinct roles in OMCS-induced pain modulation. Apart from using glutamatergic neuron specific promoter to modulate M1, using of non-specific promoter or GABAergic specific promoter in OMCS may have different outcomes which requires 
further studies. As $\alpha$-CGRP was used in our study, further studies with different neuropeptide or other molecules can be conducted to determine whether their infliction can facilitate gaining of desired outcomes by MCS. Precise temporal modulation of earmarked neuronal subtypes may guide to insights into clinically pertinent neuromodulation with less side effects and more sturdy therapeutic efficiency, moreover, insights into the cellular basis of systems-level neural circuit functioning responsible for chronic trigeminal neuropathic pain.

\section{Conclusion}

Our findings indicated that optogenetic stimulation of M1 exerts an analgesic effect in a rat model of TN. Further improvements in pain were observed following treatment with $\alpha$-CGRP, indicating that the central neuromodulation mechanism is independent of the control of peripheral pain. Optical activation of M1 via ChR2 may serve as an optical scalpel in TN. Targeted, temporally precise control of specific neural subtypes may lead to insights into clinically relevant neuromodulation with fewer side-effects and more robust therapeutic efficacy, as well as insights into the cellular basis of systems-level neural circuit function. Nonetheless, further studies are required in order to clarify the mechanisms by which optical MCS attenuates trigeminal neuropathic pain.

\section{Abbreviations}

TN: Trigeminal neuralgia; M1: Primary motor cortex; MCS: Motor cortex stimulation; DBS: Deep brain stimulation; CGRP: Calcitonin gene-related peptide; ION: Infraorbital nerve; VPM: Ventral Posteromedial nucleus of Thalamus; CCI-ION: Chronic constriction injury of ION; TNC: Trigeminal neural caudalis

\section{Acknowledgements}

Not applicable.

\begin{abstract}
Authors' contributions
Jaisan Islam and Young Seok Park were responsible for the study concept and design. Jaisan Islam was responsible for the animal experiment and surgery. Jaisan Islam and Elina KC were responsible for the electrophysiology, data analysis and immunostaining. Jaisan Islam and Young Seok Park wrote the manuscript. Byeong Ho Oh and Young Seok Park were responsible for data interpretation and manuscript review. All authors approve the final version of the manuscript.
\end{abstract}

\section{Funding}

The work was funded by the National Research Foundation of Korea (NRF, 2020R1F1A1052716). This research was financially supported by the Research Year of Chungbuk National University in 2018. This work was supported by Chungbuk National University, Chungbuk National University Hospital.

\section{Availability of data and materials}

The datasets used and/or analysed during the current study are available from the corresponding author on reasonable request.

\section{Ethics approval and consent to participate}

All experiments were approved by the Institutional Animal Care and Use Committee (IACUC) of Chungbuk National University in Korea. All animal experiments were performed during the light period at the Laboratory Animal Research Center of Chungbuk National University.

\section{Consent for publication}

Not applicable.

\section{Competing interests}

The authors have no conflicts of interest to declare.

\section{Author details}

${ }^{1}$ Department of Neuroscience, College of Medicine, Chungbuk National University, Cheongju, South Korea. ${ }^{2}$ Department of Neurosurgery, Chungbuk National University Hospital, Cheongju, South Korea. ${ }^{3}$ ISCRM, Department of Veterinary Medicine, College of Veterinary Medicine, Chungbuk National University, Cheongju, South Korea. ${ }^{4}$ Department of Neurosurgery, Chungbuk National University Hospital, College of Medicine, Chungbuk National University, 776, 1 Sunhwanro, Seowon-gu, Cheongju-Si, Chungbuk 28644, South Korea.

Received: 23 July 2020 Accepted: 17 August 2020

Published online: 26 August 2020

\section{References}

1. Henderson JM, Lad SP (2006) Motor cortex stimulation and neuropathic facial pain. Neurosurg Focus 21(6):1-4

2. Moon HC, Heo WI, Kim YJ, Lee D, Won SY, Kim HR et al (2017) Optical inactivation of the anterior cingulate cortex modulate descending pain pathway in a rat model of trigeminal neuropathic pain created via chronic constriction injury of the infraorbital nerve. J Pain Res 10:2355

3. Saavedra LC, Mendonca M, Fregni F (2014) Role of the primary motor cortex in the maintenance and treatment of pain in fibromyalgia. Med Hypotheses 83(3):332-336

4. Fontaine D, Hamani C, Lozano A (2009) Efficacy and safety of motor cortex stimulation for chronic neuropathic pain: critical review of the literature. J Neurosurg 110(2):251-256

5. Delavallee M, Rooijakkers H, Koerts G, Raftopoulos C (2011) Motor cortex stimulation in a three-year-old child with trigeminal neuropathic pain caused by a malignant glioma in the cerebellopontine angle: case report. Neurosurgery. 69(2):E494-E4E6

6. Anderson WS, Kiyofuji S, Conway JE, Busch C, North RB, Garonzik IM (2009) Dysphagia and neuropathic facial pain treated with motor cortex stimulation: case report. Neurosurgery 65(3):E626

7. Henderson JM, Boongird A, Rosenow JM, LaPresto E, Rezai AR (2004) Recovery of pain control by intensive reprogramming after loss of benefit from motor cortex stimulation for neuropathic pain. Stereotact Funct Neurosurg 82(5-6):207-213

8. Fenno L, Yizhar O, Deisseroth K (2011) The development and application of optogenetics. Annu Rev Neurosci 34:389-412

9. Kalanithi PS, Henderson JM (2012) Optogenetic neuromodulation. Int Rev Neurobiol 107: Elsevier:185-205

10. Aravanis AM, Wang L-P, Zhang F, Meltzer LA, Mogri MZ, Schneider MB et al (2007) An optical neural interface: in vivo control of rodent motor cortex with integrated fiberoptic and optogenetic technology. J Neural Eng 4(3): S143

11. Kang SJ, Kwak C, Lee J, Sim S-E, Shim J, Choi T et al (2015) Bidirectional modulation of hyperalgesia via the specific control of excitatory and inhibitory neuronal activity in the ACC. Molecular brain 8(1):81

12. Lee M, Manders TR, Eberle SE, Su C, D'amour J, Yang R et al (2015) Activation of corticostriatal circuitry relieves chronic neuropathic pain. J Neurosci 35(13):5247-5259

13. Kuzawińska O, Lis K, Cudna A, Bałkowiec-Iskra E (2014) Gender differences in the neurochemical response of trigeminal ganglion neurons to peripheral inflammation in mice. Acta Neurobiol Exp (Wars) 74(2):227-232

14. lyengar S, Ossipov MH, Johnson KW (2017) The role of calcitonin generelated peptide in peripheral and central pain mechanisms including migraine. Pain. 158(4):543

15. Kernisant M, Gear RW, Jasmin L, Vit J-P, Ohara PT (2008) Chronic constriction injury of the infraorbital nerve in the rat using modified syringe needle. J Neurosci Methods 172(1):43-47

16. Fonoff ET, Dale CS, Pagano RL, Paccola CC, Ballester G, Teixeira MJ et al (2009) Antinociception induced by epidural motor cortex stimulation in naive conscious rats is mediated by the opioid system. Behav Brain Res 196(1):63-70

17. Veinante $P$, Deschênes $M$ (2003) Single-cell study of motor cortex projections to the barrel field in rats. J Comp Neurol 464(1):98-103

18. Zilles K (1985) The cerebral cortex of the rat. A stereotaxic atlas. SpringerVerlag, New York 
19. Ding W, You Z, Shen S, Yang J, Lim G, Doheny JT et al (2017) An improved rodent model of trigeminal neuropathic pain by unilateral chronic constriction injury of distal infraorbital nerve. J Pain 18(8):899-907

20. Tang XF, Bi R, Meng Z, Gan Y (2014) Stereotaxis of mandibular nerve initial point of trigeminal ganglion in rats. Chin J Dent Res 2:99-104

21. Moon HC, Won SY, Kim EG, Kim HK, Cho CB, Park YS (2018) Effect of optogenetic modulation on entopeduncular input affects thalamic discharge and behavior in an AAV2-a-synuclein-induced hemiparkinson rat model. Neurosci Lett 662:129-135

22. DeSouza DD, Moayedi M, Chen DQ, Davis KD, Hodaie M (2013) Sensorimotor and pain modulation brain abnormalities in trigeminal neuralgia: a paroxysmal, sensory-triggered neuropathic pain. PloS One 8(6): e66340

23. Becerra L, Morris S, Bazes S, Gostic R, Sherman S, Gostic J et al (2006) Trigeminal neuropathic pain alters responses in CNS circuits to mechanical (brush) and thermal (cold and heat) stimuli. J Neurosci 26(42):10646-10657

24. Obermann M, Holle D, Katsarava Z (2011) Trigeminal neuralgia and persistent idiopathic facial pain. Expert Rev Neurother 11(11):1619-1629

25. Pinheiro AL, Marques AMC, Soares LGP, de Carvalho FB, de Oliveira SCP, Cangussú MCT (2017) The use of laser phototherapy in the management of trigeminal neuralgia pain: two decades of clinical experience. Mechanisms of Photobiomodulation Therapy XII. Sanfrancisco: International Society for Optics and Photonics

26. Kumar S, Rastogi S, Kumar S, Mahendra P, Bansal M, Chandra L (2013) Pain in trigeminal neuralgia: neurophysiology and measurement: a comprehensive review. J Med Life 6(4):383

27. Hagenacker T, Bude V, Naegel S, Holle D, Katsarava Z, Diener H-C et al (2014) Patient-conducted anodal transcranial direct current stimulation of the motor cortex alleviates pain in trigeminal neuralgia. J Headache Pain 15(1):78

28. Cowie AM, Moehring F, O'Hara C, Stucky CL (2018) Optogenetic inhibition of CGRPa sensory neurons reveals their distinct roles in neuropathic and incisional pain. J Neurosci 38(25):5807-5825

29. Holzmann B (2013) Antiinflammatory activities of CGRP modulating innate immune responses in health and disease. Curr Protein Peptide Sci 14(4): 268-274

30. Bowen EJ, Schmidt TW, Firm CS, Russo AF, Durham PL (2006) Tumor necrosis factor-a stimulation of calcitonin gene-related peptide expression and secretion from rat trigeminal ganglion neurons. J Neurochem 96(1):65-77

31. Messlinger K, Lennerz JK, Eberhardt M, Fischer MJ (2012) CGRP and NO in the trigeminal system: mechanisms and role in headache generation. Headache 52(9):1411-1427

32. Osenbach R (2006) Neurostimulation for the treatment of intractable facial pain. Pain Med 7(suppl_1):S126-SS36

33. Lefaucheur J-P, Drouot $X$, Cunin P, Bruckert $R$, Lepetit $H$, Créange $A$ et al (2009) Motor cortex stimulation for the treatment of refractory peripheral neuropathic pain. Brain. 132(6):1463-1471

34. Vaculín Š, Franěk M, Yamamotová A, Rokyta R (2008) Motor cortex stimulation in rats with chronic constriction injury. Exp Brain Res 185(2):331-335

35. Kolodziej MA, Hellwig D, Nimsky C, Benes L (2016) Treatment of central deafferentation and trigeminal neuropathic pain by motor cortex stimulation: report of a series of 20 patients. J Neurol Surg A Cent Eur Neurosurg 77(01):052-058

36. Weber K (2017) Neuromodulation and devices in trigeminal neuralgia. Headache 57(10):1648-1653

37. Thomas L, Bledsoe JM, Sandroni P, Gorman D, Lee KH (2009) Motor cortex and deep brain stimulation for the treatment of intractable neuropathic face pain. Curr Neurol Neurosci Rep 9(2):120

38. Behrens TE, Johansen-Berg H, Woolrich M, Smith S, Wheeler-Kingshott C Boulby P et al (2003) Non-invasive mapping of connections between human thalamus and cortex using diffusion imaging. Nat Neurosci 6(7):750

39. Sanes JN, Donoghue JP (2000) Plasticity and primary motor cortex. Annu Rev Neurosci 23(1):393-415

40. Bachmann CG, Muschinsky S, Nitsche MA, Rolke R, Magerl W, Treede R-D et al (2010) Transcranial direct current stimulation of the motor cortex induces distinct changes in thermal and mechanical sensory percepts. Clin Neurophysiol 121(12):2083-2089

41. B-c S, D-r K, H-s K, Lee S-w (2014) Simultaneous trial of deep brain and motor cortex stimulation in chronic intractable neuropathic pain. Stereotact Funct Neurosurg 92(4):218-226
42. Monsalve GA (2012) Motor cortex stimulation for facial chronic neuropathic pain: a review of the literature. Surg Neurol Int 3(Suppl 4):S290

43. Rainov NG, Heidecke V (2003) Motor cortex stimulation for neuropathic facial pain. Neurol Res 25(2):157-161

44. DosSantos MF, Ferreira N, Toback RL, Carvalho AC, DaSilva AF (2016) Potential mechanisms supporting the value of motor cortex stimulation to treat chronic pain syndromes. Front Neurosci 10:18

45. Hodaj H, Alibeu J-P, Payen J-F, Lefaucheur J-P (2015) Treatment of chronic facial pain including cluster headache by repetitive transcranial magnetic stimulation of the motor cortex with maintenance sessions: a naturalistic study. Brain Stimul 8(4):801-807

46. Katayama Y, Fukaya C, Yamamoto T (1998) Poststroke pain control by chronic motor cortex stimulation: neurological characteristics predicting a favorable response. J Neurosurg 89(4):585-591

47. Khodashenas M, Towhidkhah F, Baghdadi G (2018) A conceptual model of trigeminal Neuralgia network and tDCS pain reduction effect, vol 01

48. Pagano RL, Fonoff ET, Dale CS, Ballester G, Teixeira MJ, Britto LR (2012) Motor cortex stimulation inhibits thalamic sensory neurons and enhances activity of PAG neurons: possible pathways for antinociception. Pain 153(12):2359-2369

49. Reidler JS, Mendonca ME, Santana MB, Wang X, Lenkinski R, Motta AF et al (2012) Effects of motor cortex modulation and descending inhibitory systems on pain thresholds in healthy subjects. J Pain 13(5):450-458

50. Anderson CT, Sheets PL, Kiritani T, Shepherd GM (2010) Sublayer-specific microcircuits of corticospinal and corticostriatal neurons in motor cortex. Nat Neurosci 13(6):739

51. Kleinlogel S, Feldbauer K, Dempski RE, Fotis H, Wood PG, Bamann C et al (2011) Ultra light-sensitive and fast neuronal activation with the Ca $2+$ -permeable channelrhodopsin CatCh. Nat Neurosci 14(4):513

52. Beaudry H, Daou I, Ase AR, Ribeiro-da-Silva A, Séguéla P (2017) Distinct behavioral responses evoked by selective optogenetic stimulation of the major TRPV1+ and MrgD+ subsets of C-fibers. Pain. 158(12):2329-2339

53. Zhang F, Wang L-P, Boyden ES, Deisseroth K (2006) Channelrhodopsin-2 and optical control of excitable cells. Nat Methods 3(10):785

54. Zhang Y-P, Oertner TG (2007) Optical induction of synaptic plasticity using a light-sensitive channel. Nat Methods 4(2):139

55. Nagel G, Szellas T, Huhn W, Kateriya S, Adeishvili N, Berthold P et al (2003) Channelrhodopsin-2, a directly light-gated cation-selective membrane channel. Proc Natl Acad Sci 100(24):13940-13945

56. Gunaydin LA, Yizhar O, Berndt A, Sohal VS, Deisseroth K, Hegemann P (2010) Ultrafast optogenetic control. Nat Neurosci 13(3):387

57. Gradinaru V, Thompson KR, Zhang F, Mogri M, Kay K, Schneider MB et al (2007) Targeting and readout strategies for fast optical neural control in vitro and in vivo. J Neurosci 27(52):14231-14238

58. Liu S, Tao F (2016) Application of optogenetics-mediated motor cortex stimulation in the treatment of chronic neuropathic pain. J Transl Sci 2(5): 286-288

59. Guy N, Chalus M, Dallel R, Voisin DL (2005) Both oral and caudal parts of the spinal trigeminal nucleus project to the somatosensory thalamus in the rat. Eur J Neurosci 21(3):741-754

60. Idänpään-Heikkilä JJ, Guilbaud G (1999) Pharmacological studies on a rat model of trigeminal neuropathic pain: baclofen, but not carbamazepine, morphine or tricyclic antidepressants, attenuates the allodynia-like behaviour. Pain 79(2-3):281-290

61. Leiser SC, Moxon KA (2006) Relationship between physiological response type (RA and SA) and vibrissal receptive field of neurons within the rat trigeminal ganglion. J Neurophysiol 95(5):3129-3145

62. Pagano RL, Assis DV, Clara JA, Alves AS, Dale CS, Teixeira MJ et al (2011) Transdural motor cortex stimulation reverses neuropathic pain in rats: a profile of neuronal activation. Eur J Pain 15(3):268. e1-268.e14

63. Tseng W-T, Tsai M-L, Iwata K, Yen C-T (2012) Long-term changes in trigeminal ganglionic and thalamic neuronal activities following inferior alveolar nerve transection in behaving rats. J Neurosci 32(45):16051-16063

\section{Publisher's Note}

Springer Nature remains neutral with regard to jurisdictional claims in published maps and institutional affiliations. 